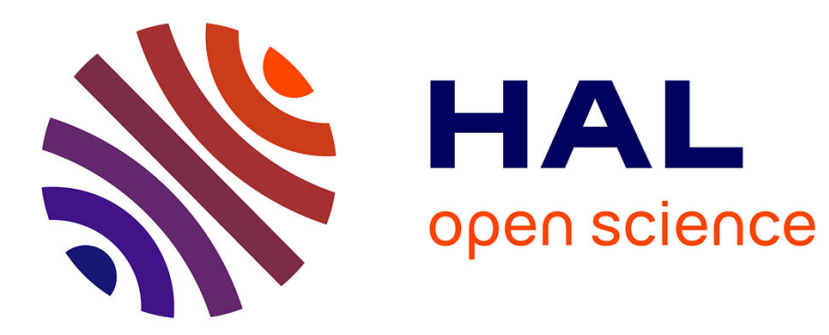

\title{
Minimizing Input Torques of a High-Speed Five-Bar Mechanism by using Variable Stiffness Springs
}

Rafael Balderas Hill, Sébastien Briot, Abdelhamid Chriette, Philippe Martinet

\section{To cite this version:}

Rafael Balderas Hill, Sébastien Briot, Abdelhamid Chriette, Philippe Martinet. Minimizing Input Torques of a High-Speed Five-Bar Mechanism by using Variable Stiffness Springs. 22nd CISM IFToMM Symposium on Robot Design, Dynamics and Control (RoManSy 2018), Jun 2018, Rennes, France. hal-01759341

\author{
HAL Id: hal-01759341 \\ https://hal.science/hal-01759341
}

Submitted on 24 Jun 2019

HAL is a multi-disciplinary open access archive for the deposit and dissemination of scientific research documents, whether they are published or not. The documents may come from teaching and research institutions in France or abroad, or from public or private research centers.
L'archive ouverte pluridisciplinaire HAL, est destinée au dépôt et à la diffusion de documents scientifiques de niveau recherche, publiés ou non, émanant des établissements d'enseignement et de recherche français ou étrangers, des laboratoires publics ou privés. 


\title{
Minimizing Input Torques of a High-Speed Five-Bar Mechanism by using Variable Stiffness Springs
}

\author{
Rafael Balderas Hill $\dagger^{*}$, Sébastien Briot $\stackrel{\ddagger}{*}^{*}$, Abdelhamid Chriette ${ }^{{ }^{*}}$, and \\ Philippe Martinet ${ }^{*}$ \\ † Ecole Centrale de Nantes, 44321 Nantes, France \\ $¥$ Centre National de la Recherche Scientifique (CNRS) \\ ${ }^{*}$ Laboratoire des Sciences du Numérique de Nantes (LS2N), UMR CNRS 6004, \\ 44321 Nantes, France \\ $\S$ Centre de Recherche Inria Sophia Antipolis, 06902 Sophia Antipolis, France
}

\begin{abstract}
Recently, variable stiffness actuators (VSAs) have been introduced for reducing the input efforts of pick-and-place robots. However, the serial arrangement of springs and motors in the VSAs decreases the accuracy at high-speeds due to uncontrolled robot deflections. To ensure accuracy while reducing the input efforts, this paper proposes the use of variable stiffness springs (VSS) in parallel configuration with the motors. The parallel arrangement of VSS and motors is combined with a shooting method to adjust the stiffness of the system in order to enforce its limit cycle to converge to a desired trajectory, and, thus, to decrease the input torques. Numerical simulations of the suggested approach on a fivebar mechanism show the reduction of the robot input efforts.
\end{abstract}

\section{Introduction}

It is well-known that one of the most common operations for high-speed robot manipulators in industrial applications is to pick and place objects from one initial desired position to a final position. Typically, such types of operations are fast pseudo-periodic motions, with variable amplitudes and frequencies in which an enormous amount of input efforts is required to move and stop the robot in the pick and place positions. As a consequence, a large quantity of energy is required to make the robot move, and then this energy must be dissipated to stop the robot in the braking phase. This is costly in terms of energy efficiency.

The classical approach to reduce the input torques at high speeds is by lowering the moving elements mass of the robot architecture, thus permitting the use of less powerful motors [1]. Even if this allows reducing the 
input torques, the lightweight structure affects the robot stiffness, leading to decrease the accuracy of the mechanism.

To operate at slow speeds, gravity-balancing techniques [2][3] have shown their effectiveness to compensate the input efforts required to move the links of slow pick-and-place robots. However, these techniques cannot be applied for high-speed robots for which the inertial effects are preponderant. Thus, in the last years, several researchers have proposed the use of Variable Stiffness Actuators (VSAs) to minimize the input efforts of pick-and-place robots [4][5]. The main idea is to connect the motor and the output link by a spring in series, which serves as energy storage and whose stiffness is controllable in order to adapt to different tasks. These springs are called Variable Stiffness Springs (VSS)[6]. Nevertheless, the main issue is that the serial arrangement of VSS and motors in the VSAs leads to uncontrolled robot deflections at high-speeds, worsening the accuracy of the robot.

A novel type of actuation, for a slow parallel robot in [7] and a slow serial robot in [8][9], proposes the use constant stiffness springs in parallel to the motors in order to reduce the input torques in pick-and-place operations. Even if the results are impressive, the level of compliance to adapt to fast pseudo-periodic pick-and-place motions is limited due to the fixed stiffness.

Therefore, the aim of this paper is to propose a strategy for reducing the input efforts of a high-speed five-bar mechanism by placing VSS in parallel to the motors. Furthermore, we deal with the minimization of input torques by exploiting its dynamic model, by finding the optimal stiffness, such that the limit cycle of the system converges to the desired pseudo-periodic pickand-place motions. Then, the optimal stiffness is adjusted by means of the VSS. By adding a spring with controllable stiffness in parallel to the five-bar mechanism actuated links, two performances are achieved: i) direct power connection between the motor and the robot links, thus ensuring accuracy at high-speeds; ii) control of the stored potential energy to be released per cycle of the pick-and-place motion. In order to show the effectiveness of the proposed approach, two cases are compared and analyzed:

- Nominal case, which consists of the parallel robot without springs;

- Use of VSS in parallel with the motors of the robot with the proposed limit cycle tracking algorithm.

\section{Physical Background}

\subsection{Dynamic model of five-bar mechanism with variable stiffness springs in parallel configuration with the actuated links}

The general scheme of the five-bar mechanism with variable stiffness springs in parallel is given in Fig. 1. The actuation of the parallel robot is 


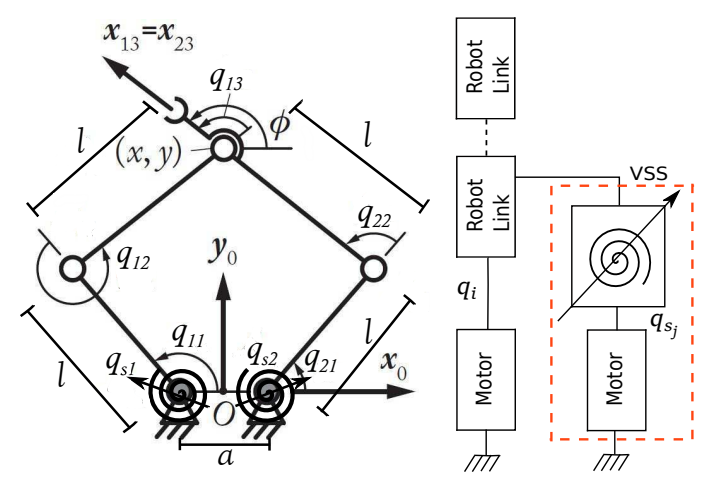

Figure 1. On the left the five-bar mechanism parameterization with two actuated joints $q_{11}$ and $q_{21}$, and three passive joints $q_{12}, q_{22}$ and $q_{13}$ is shown. The variable stiffness torsional springs are located in parallel to the two actuated joints defined by $q_{s 1}$ and $q_{s 2}$. On the right the power transmission system of VSS in parallel to the motors is shown. $q_{i}$ and $q_{s_{j}}$ represent the parallel robot joints and variable stiffness joints coordinates, respectively, and $i=1, \ldots, 2, j=1, \ldots, 2$.

provided by $\mathbf{q}=\left[q_{11}, q_{21}\right]^{T}$. The vector of passive joints is given by $\mathbf{q}_{d}=$ $\left[q_{12}, q_{22}, q_{13}\right]^{T}$. The vector of moving platform pose is given by $\mathbf{x}=[x, y]^{T}$. $a$ is the distance between the actuated joints. All link lengths $l$ are identical. As shown in Fig. 1, the actuation for the variable stiffness joints is given by $\mathbf{q}_{s}=\left[q_{s 1}, q_{s 2}\right]^{T}$. For the dynamic modeling of the five-bar mechanism, the model of a real robot called DexTAR [10] was used. DexTAR is a five-bar mechanism whose dynamic model is computed in [10] using the Lagrange formalism. Adapting its dynamic model to our purpose, by considering the effects of the elastic deformation of the VSS in parallel with the actuated links (Fig. 1), the DexTAR dynamics computed in [10] become:

$$
\boldsymbol{\tau}=\mathbf{Z Z} \ddot{\mathbf{q}}+m \mathbf{J}^{T} \ddot{\mathbf{x}}+\boldsymbol{\tau}_{s}
$$

where

- $\mathbf{J}$ is the robot Jacobian matrix;

- $\tau$ is the two-dimensional vector of robot input efforts;

- $\boldsymbol{\tau}_{s}$ is the two-dimensional vector of elastic torques associated to the VSS coupled to the robot in parallel by the following expression:

$$
\boldsymbol{\tau}_{s}=\mathbf{K}\left(\mathbf{q}-\mathbf{q}_{s}\right)
$$

in which $\mathbf{K}=\operatorname{diag}\left(k_{11}, k_{21}\right)$, with $k_{11}, k_{21}$ being the springs constants; - $m$ is the mass of the end-effector. 
- $\mathbf{Z Z}=\operatorname{diag}\left(z z_{11}, z z_{21}\right)$, with $z z_{11}$ and $z z_{21}$ representing the inertia of the two proximal links.

In order to express the dynamic model in terms of the joint space accelerations, $\ddot{\mathrm{x}}$ in (1) is mapped into the joint space by using the second-order kinematic constraint relations [11]:

$$
\ddot{\mathbf{x}}=\mathbf{J} \ddot{\mathbf{q}}+\mathbf{b}
$$

where $\mathbf{b}$ is a vector containing terms in squared velocities [10]. Skipping all mathematical derivations, we get

$$
\boldsymbol{\tau}=\left(\mathbf{Z Z}+m \mathbf{J}^{T} \mathbf{J}\right) \ddot{\mathbf{q}}+m \mathbf{J}^{T} \mathbf{b}+\mathbf{K}\left(\mathbf{q}-\mathbf{q}_{s}\right)
$$

Finally, the VSS dynamics is given by:

$$
\boldsymbol{\tau}_{v s s}=\mathbf{J} \ddot{\mathbf{q}}_{s}-\mathbf{K}\left(\mathbf{q}-\mathbf{q}_{s}\right)
$$

where $\mathbf{J}=\operatorname{diag}\left(J_{1}, J_{2}\right)$, with $J_{1}$ and $J_{2}$ being the inertias of the couplings between the motors and the springs.

Based on the aforementioned computations, it is necessary to find a strategy to exploit the dynamic model of the robot in order to vanish the input torques and let the springs, whose stiffness is controlled by $\mathbf{q}_{s}$, to supply most of the energy to move the links of the robot.

\section{Control of pick-and-place oscillations}

For systems that oscillate at a constant amplitude and frequency, it is possible to find a unique set of spring constants grouped in matrix $\mathbf{K}$, such that the limit cycle of the system converge to the desired periodic trajectory, thus vanishing the input torques [7][9]. However, it is not the same case for pick-and-place operations, which are typically defined by pseudo-periodic oscillatory motions, where the amplitudes and frequencies of oscillation may vary. This is mainly due to the fact that for each pseudo-frequency, there exist a different set of spring constants for enforcing the limit cycle to converge to the desired pseudo-periodic trajectory. That is why, similar to what it was done in [8], we apply the so-called shooting method to find the optimal spring coordinates $\mathbf{q}_{s}$ that adjust the output stiffness, so that the limit cycle matches as best as possible the desired trajectory even if the motion is quasi-periodic. This allows to have a fixed set of spring constants for any pseudo-frequency, and by tuning $\mathbf{q}_{s}$ we ensure the convergence of (1) to an unforced second order differential equation, thus decreasing the input torques $\boldsymbol{\tau}$. As shown in Fig. 2, for performing energy-efficient pickand-place motions, the main idea is to tune the stiffness variables $\mathbf{q}_{s}$ when 


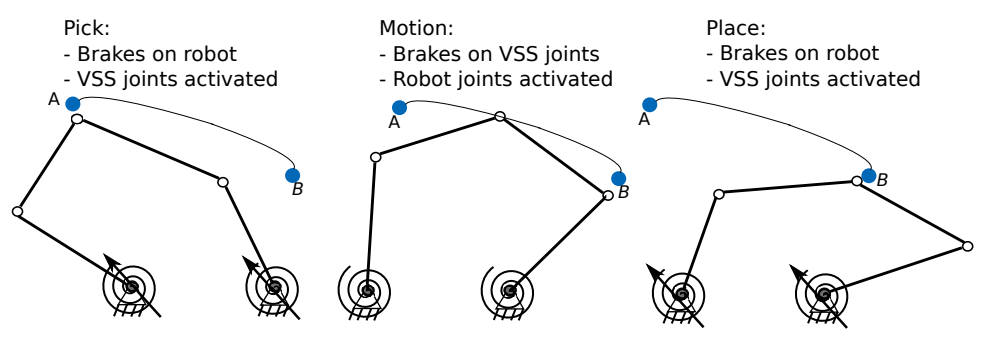

Figure 2. Five-bar mechanism in the three operation modes: In pick and place position the robot is stopped and the VSS is adjusted through $\mathbf{q}_{s}$ to match the limit cycle to oscillate from $A$ to $B$. Then in the motion phase the VSS is stopped and the robot is controlled only by $\mathbf{q}$.

the robot is stopped in a pick or place position. This to ensure meeting the desired boundary conditions (pick or place points) of the next cycle at a given pseudo-frequency. The shooting method formulation applied to find the spring coordinates $\mathbf{q}_{s}$ seeks to solve (4) for $\boldsymbol{\tau}=\mathbf{0}$, i.e.:

$$
\ddot{\mathbf{q}}=-\left(\mathbf{Z Z}+m \mathbf{J}^{T} \mathbf{J}\right)^{-1}\left(m \mathbf{J}^{T} \mathbf{b}+\mathbf{K}\left(\mathbf{q}-\mathbf{q}_{s}\right)\right)
$$

with the boundary conditions defined as the desired pick and place positions and velocities by $\mathbf{q}(0)=\mathbf{q}_{0}^{*}, \mathbf{q}\left(t_{f}\right)=\mathbf{q}_{t_{f}}^{*}, \dot{\mathbf{q}}(0)=\dot{\mathbf{q}}_{0}^{*}$ and $\dot{\mathbf{q}}\left(t_{f}\right)=\dot{\mathbf{q}}_{t_{f}}^{*}$ by formulating the following position and velocity error vectors, respectively:

$$
\begin{aligned}
& \mathbf{E}_{q}\left(\mathbf{q}_{s}\right)=\mathbf{q}\left(t_{f}, \mathbf{q}_{s}\right)-\mathbf{q}_{t_{f}}^{*} \\
& \mathbf{E}_{\dot{q}}\left(\mathbf{q}_{s}\right)=\dot{\mathbf{q}}\left(t_{f}, \mathbf{q}_{s}\right)-\dot{\mathbf{q}}_{t_{f}}^{*}
\end{aligned}
$$

where $t_{f}$ is the final time, and $\dot{\mathbf{q}}$ and $\mathbf{q}$ are obtained from numerical single and double integration of $\ddot{\mathbf{q}}$, respecitvely. Thus, the shooting method is applied to iteratively find the spring coordinates $\mathbf{q}_{s}$, such that expressions (7) and (8) converge to zero as follows:

$$
\begin{aligned}
& \text { while } \mathbf{E}_{q}>\boldsymbol{\epsilon}_{1}, \mathbf{E}_{\dot{q}}>\boldsymbol{\epsilon}_{2}, k \leq \max _{k} \text { do } \\
& \qquad \begin{array}{l}
\mathbf{E}_{q}\left(\mathbf{q}_{s}\right)=\mathbf{q}\left(t_{f}, \mathbf{q}_{s}\right)-\mathbf{q}_{t_{f}}^{*} \\
\mathbf{E}_{\dot{q}}\left(\mathbf{q}_{s}\right)=\dot{\mathbf{q}}\left(t_{f}, \mathbf{q}_{s}\right)-\dot{\mathbf{q}}_{t_{f}}^{*} \\
\mathbf{q}_{s_{k}}:\left[\mathbf{E}_{q}\left(\mathbf{q}_{s_{k}}\right), \mathbf{E}_{\dot{q}}\left(\mathbf{q}_{s_{k}}\right)\right]=0 \\
\mathbf{q}_{s_{k+1}}=\mathbf{q}_{s_{k}}+\Delta \mathbf{q}_{s_{k}}
\end{array} \\
& \text { end }
\end{aligned}
$$

Optimal set of VSS coordinates: $\mathbf{q}_{s}=\mathbf{q}_{s_{k+1}}$

Algorithm 1: Shooting method applied to limit cycle tracking by updating spring coordinates $\mathbf{q}_{s}$

where $\max _{k}$ is the maximum number of iterations, $\boldsymbol{\epsilon}_{1}$ and $\boldsymbol{\epsilon}_{2}$ are error thresholds. 


\section{Simulation Results}

The proposed approach for minimizing the input torques was validated by using the following numerical values for the five-bar mechanism geometric and dynamic parameters: inertia of the two proximal links $z z_{11}=0.0133$ $\mathrm{kg} / \mathrm{m}^{2}$ and $z z_{21}=0.0142 \mathrm{~kg} / \mathrm{m}^{2}$, end-effector mass $m=0.537 \mathrm{~kg}$, inertias of the couplings between the motors and springs $J_{1}=J_{2}=0.0051 \mathrm{~kg} / \mathrm{m}^{2}$, stiffness constants of the springs $k_{11}=k_{21}=10 \mathrm{Nm} / \mathrm{rad}$, link lengths $l=0.090 \mathrm{~m}$, distance between the axes of the actuated links $a=0.118$ $\mathrm{m}$. The limit cycle tracking (Algorithm 1) proposed in section 3 was tested by defining multiple desired points in the five-bar mechanism workspace as shown in Fig. 3. Each segment from the multiple-point pick-and-place sequence has a different travel time in order to show the effectiveness of the proposed approach to reduce the efforts in quasi-periodic motions. Moreover, in order to make the comparisson between the nominal actuation and the actuation with VSS, the total travel time of the nominal case is set equal to the travel-plus-adjusting times of the actuation with VSS.

Table 1 shows the optimal values obtained from the algorithm described in section 3. It can be seen that for every segment of trajectory there is a different total time, i.e. travel plus adjusting times, and therefore, different optimal values for the VSS coordinates $\mathbf{q}_{s}$. Table 2 shows the values of the maximum torques for the different segments for the two types of actuation: nominal without VSS and with VSS in parallel to the actuated links of the five-bar mechanism. For the second type of actuation the input efforts from the full actuation chain are computed, i.e. the robot torques and the VSS input torques. It can be seen that the average torque reduction from the full actuation chain, for the full multiple-point pick-and-place sequence, based on the maximum values of the torques from Table 2 , is of $53 \%$. It is also possible to observe that the variable stiffness joint torques grouped in $\tau_{s}$ provide most of the effort in the full actuation chain.

Table 1. Optimal values for the VSS coordinates $q_{s 1}$ and $q_{s 2}$ for every segment of trajectory.

\begin{tabular}{|l|l|l|l|}
\hline Segment & $\begin{array}{l}\text { Total time (Travel } \\
+ \text { Adjusting) }(\mathrm{s})\end{array}$ & Optimal $q_{s_{1}}(\mathrm{rad})$ & Optimal $q_{s_{2}}(\mathrm{rad})$ \\
\hline$A \rightarrow B$ & 0.1 & 0.688 & 0.499 \\
\hline$B \rightarrow C$ & 0.09 & -0.844 & -1.210 \\
\hline$C \rightarrow D$ & 0.12 & 0.387 & 0.560 \\
\hline$D \rightarrow E$ & 0.07 & -1.421 & -1.417 \\
\hline
\end{tabular}




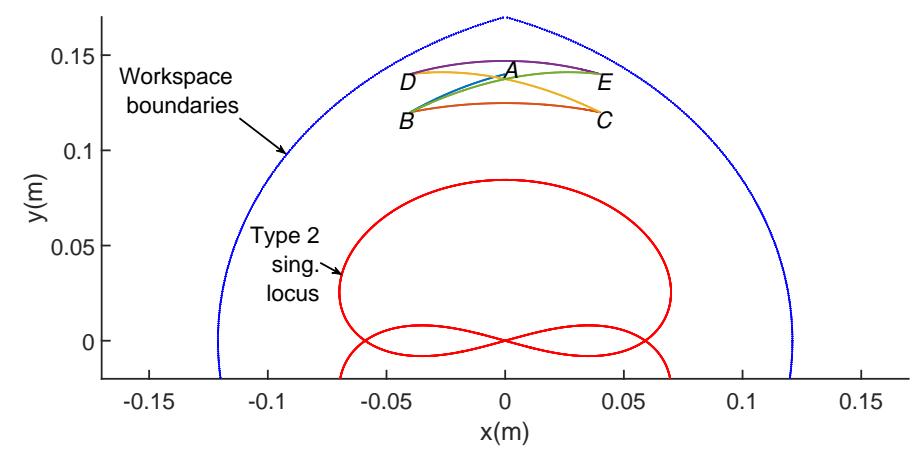

Figure 3. Multiple-point pick-and-place sequence in the five-bar mechanism workspace: $A \rightarrow B$ (travel time: $0.1 \mathrm{~s}$ ), $B \rightarrow C$ (travel time: $0.09 \mathrm{~s}$ ), $C \rightarrow$ $D$ (travel time: $0.12 \mathrm{~s}$ ), $D \rightarrow E$ (travel time: $0.07 \mathrm{~s}$ ).

Table 2. Maximum values of input torques for nominal case which consists of the five-bar mechanism without springs, and input torques by using VSS in parallel with same adjusting times as travel times.

\begin{tabular}{|c|c|c|c|c|}
\hline & & $\begin{array}{c}\text { Nominal } \\
\text { Max. Torques }\end{array}$ & \multicolumn{2}{c|}{$\begin{array}{c}\text { Using VSS in parallel } \\
\text { Max. Torques (Full actuation) }\end{array}$} \\
\hline Segment & Time (s) & $|\boldsymbol{\tau}|_{\max }(\mathrm{Nm})$ & $|\boldsymbol{\tau}|_{\max }(\mathrm{Nm})$ & $\left|\boldsymbol{\tau}_{\text {vss }}\right|_{\max }(\mathrm{Nm})$ \\
& & & & \\
\hline$A \rightarrow B$ & 0.1 & {$[5.451,4.125]$} & {$[0.177,0.187]$} & {$[2.546,1.849]$} \\
\hline$B \rightarrow C$ & 0.09 & {$[11.599,12.811]$} & {$[0.204,0.207]$} & {$[4.996,5.698]$} \\
\hline$C \rightarrow D$ & 0.12 & {$[5.408,8.381]$} & {$[0.146,0.202]$} & {$[2.654,3.946]$} \\
\hline$D \rightarrow E$ & 0.07 & {$[20.544,20.956]$} & {$[0.195,0.201]$} & {$[8.472,8.980]$} \\
\hline
\end{tabular}

\section{Conclusions and Future Works}

In this paper, we have presented a new approach to minimize the input efforts of a high-speed five-bar mechanism, based on the use VSS in parallel to the motors, combined with a limit cycle tracker. This has allowed to considerably reduce the input torques of fast quasi-periodic motions. Simulations led to a successful minimization of the input torques for different multiplepoint trajectories on a five-bar mechanism by analyzing two cases: nominal without springs and VSS with adjustment of the limit cycles. Results show that the input torques reduction of the full actuation chain can reach up to $53 \%$ considering also the efforts from the motors that adjust the stiffness. Future work on this subject includes experimental validation of our approach and the optimal design of an energy-efficient five-bar mechanism with the VSS in parallel. 


\section{Acknowledgment}

This work was conducted with the support of the Mexican Council for Science and Technology (CONACYT).

\section{Bibliography}

[1] R. Clavel: Device for the movement and positioning of an element in space. US Patent 4976 582, December 1990

[2] S. Briot and V. Arakelian: A New Energy-free Gravity-compensation Adaptive System for Balancing of 4-DOF Robot Manipulators with Variable Payloads. In Proc. of the 14th IFToMM World Congress (2015 IFToMM World Congress), October 2015, Taipei, Taiwan.

[3] S. Briot, V. Arakelian, N. Sauvestre and J.-P. Le Baron. Shaking Forces Minimization of High-Speed Robots via an Optimal Motion Planning. In Proc. of the 18th CISMIFToMM Symposium on Robot Design, Dynamics, and Control (ROMANSY 2010), July 2010, Udine, Italy.

[4] M. Uemura and S. Kawamura: Resonance-based motion control method for multijoint robot through combining stiffness adaptation and iterative learning control. In IEEE International Conference on Robotics and Automation, Kobe, Japan, pp. 1543-1548, 2009.

[5] H. Goya, K. Matsusaka, M. Uemura, Y. Nishioka, and S. Kawamura: Realization of high-energy efficient pick-and-place tasks of scara robots by resonance. In IEEE/RSJ International conference on Intelligent Robots and Systems, IROS 2012, pp. 27302735, October 2012, Vilamoura, Algarve (Portugal).

[6] K. Hollander and T. Sugar. Concepts for compliant actuation in wearable robotic systems. In Proc. of the US-Korea Conference on Science, Technology and Entrepreneurship (UKC04), 2004, vol. 128, pp. 644-650.

[7] J.P. Barreto, F.J.-F. Schöler and B. Corves: The concept of natural motion for pick and place operations. In: New advances in mechanisms, mechanical transmissions and robotics. Springer, pp. 89-98.

[8] W. Schiehlen, and N. Guse: Control of Limit Cycle Oscillations, In Proc. of the IUTAM Symposium on Chaotic Dynamics and Control of Systems and Processes in Mechanics, pp. 429-439, 2005.

[9] W. Schiehlen, and M. Iwamura, Minimum Energy Control of Multibody Systems Utilizing Storage Elements, In Proc. of DETC 2009, ASME DETC 2009 86327, 2009.

[10] S. Briot, N. Bouton and P. Bigras: Controlling Parallel Robots during Singular Assembly Mode Changing. In Proc. of the 4th Joint International Conference on Multibody System Dynamics. Montreal, Canada, May 2016.

[11] J-P. Merlet: Parallel Robots. Springer, 2006. ISBN 978-1-4020-4133-4. 